\section{Nomenclature}

$=$ area of the mass transfer electrode

$C_{b} \quad=$ bulk concentration

$\mathscr{D} \quad=$ molecular diffusivity

$F \quad=$ Faraday's constant

$H_{\mathrm{av}} \quad=$ average spacing between wavy walls

$i_{d} \quad=$ diffusional current

$k \quad=$ mass transfer coefficient

$L \quad=$ mass transfer length

$Q \quad=$ volumetric flow rate

Re $\quad=$ Reynolds number $\left(=H_{\mathrm{av}} u_{\mathrm{av}} / v\right)$

$R e_{c s} \quad=$ critical Reynolds number for straight channel $(=2000)$

$R e_{c w} \quad=$ critical Reynolds number for wavy channel $(=350)$

$=$ Schmidt number

$=$ cycle-average Sherwood number $\left(=k H_{\mathrm{av}} / \mathscr{D}\right)$

$=$ Sherwood number for straight channel

= Sherwood number for wavy channel

$=$ local Sherwood number

$=$ local wall velocity gradient

$=$ velocity based on $H_{\mathrm{av}}\left(=Q / W H_{\mathrm{av}}\right)$

$=$ width of wavy wall

$=$ distance of streamwise direction

= number of electrons exchanged $\lambda \quad=$ wave length

$=$ kinematic viscosity

$[\mathrm{m}]$

$\left[\mathrm{m}^{2} / \mathrm{s}\right]$ $\left[\mathrm{kg}-\mathrm{mol} / \mathrm{m}^{3}\right.$

$\left[\mathrm{m}^{2} / \mathrm{s}\right]$

$[\mathrm{C} / \mathrm{kg}-\mathrm{mol}]$

$[\mathrm{m}]$

$[\mathrm{A}]$

$[\mathrm{m} / \mathrm{s}]$

$[\mathrm{m}]$

$\left[\mathrm{m}^{3} / \mathrm{s}\right]$

$[-]$

$[-]$

$[-]$

$[-]$

$[-]$

$[-]$

$[-]$

$[-]$

$[1 / \mathrm{s}]$

$[\mathrm{m} / \mathrm{s}]$

[m]

$[\mathrm{m}]$

Literature Cited

1) Bellhouse, B. J., F. H. Bellhouse, C. M. Curl, T. I. MacMillan, A. J. Gunning, E. H. Spratt, S. B. Macmurray and J. M. Nelems: Trans. Am. Soc. Artif. Int. Organs., 19, 72 (1973).

2) Chow, J. C. F. and K. Soda: Trans. ASME, J. of Heat Transfer, 95, 352 (1973).

3) Fedkiw, P. and J. Newman: AIChE J., 25, 1077 (1979).

4) Fedkiw, P. and J. Newman: AIChE J., 23, 255 (1977).

5) Mackley, N. V.: Ph. D. Thesis, Univ. of Aston in Birmingham (1973).

6) Mendes, P. S. and E. M. Sparrow: Trans. ASME, J. of Heat Transfer, 106, 55 (1984)

7) Mizushina, T.: Advances in Heat Transfer, 7, 87 Academic Press (1977).

8) Nishimura, T. and Y. Kawamura: Kagaku Kogaku Ronbunshu, 7, 469 (1981); Heat Transfer Japanese Research, 10, 82 (1981).

9) Nishimura, T., Y. Ohori and Y. Kawamura: J. Chem. Eng. Japan, 17, 466 (1984).

10) Saito, T. and $Y$. Ito: Preprints of 18 th National Heat Transfer Symposium of Japan, 277 (1981).

11) Sparrow, E. M. and.A. T. Prata: Numerical Heat Transfer, 6, 441 (1983).

\title{
EQUATION OF STATE BASED ON A GROUP CONTRIBUTION MODEL APPLICABLE TO VAPOR AND LIOUID PHASES AND ITS APPLICATION TO $n$-ALKANE SYSTEMS
}

\author{
SHIGEKI TAKISHIMA AND SHOZABURO SAITO \\ Department of Chemical Engineering, Tohoku University, Sendai 980
}

Key Words: Equation of State, Group Contribution Model, Hole Theory, Nonrandom Distribution Model, Vapor-Liquid Equilibrium, Normal Alkane

\begin{abstract}
An equation of state is derived from a group contribution model which takes into account the effect of local molecular distribution on potential energy in terms of the non-random two-liquid (NRTL) model. Model parameters for methyl and methylene groups are determined from the data reduction of saturated properties of $\boldsymbol{n}$ alkanes. It is shown that the equation of state correlates well the saturated properties of $n$-alkanes and successfully predicts the vapor-liquid equilibria of $n$-alkane binary mixtures.
\end{abstract}

\section{Introduction}

Group contribution models constitute a method for predicting thermodynamic quantities from the properties of the common structural units of molecules. The development of group contribution models will be of advantage to the prediction of the properties of

Received June 7, 1985. Correspondence concerning this article should be addressed to S. Saito. molecules of which only the molecular structure may be known. In particular, it is believed that the development of group contribution models will make prediction possible for polymer systems as well as for systems of molecules for which critical point data are not available.

Many thermodynamic quantities can be calculated from an equation of state. There are two ways of applying the group contribution models, in relation to 
equations of state. One is to estimate the parameters appearing in empirical equations of state when applied to mixtures. ${ }^{14)}$ The other is to develop an equation of state on the basis of a group contribution model. Although early successful approaches of this kind were reported by Chao and his coworkers, ${ }^{6,8)}$ their models can be applied only to calculate liquidphase properties. Most equations of state based on group contribution models do not satisfy the ideal gas limit at infinite volume and are not applicable to the vapor phase.

Recently a few equations of state based on group contribution models have been proposed which satisfy the ideal gas limit. Skjold-Jørgensen ${ }^{13)}$ derived an equation of state from the van der Waals partition function. A group contribution method was applied to estimate the parameters of their equation. Another equation of state was proposed by one of the authors. ${ }^{5)}$ In the derivation of this equation, a group contribution model combined with hole theory was used to develop a form of the partition function. A comparison between experimental and calculated results showed the efficiency of this equation of state in correlating and predicting thermodynamic properties: liquid molar volumes, heats of vaporization and activity coefficients. However, predictions for saturated vapor pressures and vapor molar volumes were not satisfactory.

The purpose of this work is to derive an equation of state based on a group contribution model applicable to the estimation of the saturated properties. A developed model has been obtained from a modification of our previous one.

\section{Development of Equation of State}

In the previous model, ${ }^{5}$ three main assumptions were made: 1) the combinatorial factor was approximated by the Flory-Huggins' expression, ${ }^{2,4)}$ 2) the free volume was expressed by a simplified Henderson's approximation, ${ }^{37}$ and 3) total potential energy was estimated assuming a random distribution of segments. On these assumptions, the following equation of state was finally derived:

$$
\frac{P V}{N k T}=1-r-\frac{V}{V_{0}} \ln \frac{V-V_{0} r}{V}+\frac{c r V_{0}}{V-V_{0} r}-\frac{V_{0} T^{*}}{V T}
$$

where

$$
\begin{array}{rlrl}
N & =\sum_{i} N_{i} \\
V_{0} & =N v_{0} & \\
r & =\sum_{i} x_{i} r_{i}, \quad r_{i}=\sum_{s} v_{i}^{(s)} r^{(s)} \\
c & =\sum_{i} x_{i} c_{i}, \quad c_{i}=\sum_{s} v_{i}^{(s)} r^{(s)} c^{(s)} \\
x_{i} & =N_{i} / N &
\end{array}
$$

$$
\begin{aligned}
T^{*} & =(z / 2) \sum_{s} \sum_{i} w^{(s)} w^{(t)} U^{(t s)} \\
w^{(s)} & =\left(\sum_{i} N_{i} v_{i}^{(s)} r^{(s)}\right) / N_{T} \\
N_{T} & =N_{0}+\sum_{i} \sum_{s} N_{i} v_{i}^{(s)} r^{(s)}=N_{0}+N r \\
U^{(t s)} & =C_{U 1}^{(t s)}+C_{U 2}^{(t s)} T+C_{U 3}^{(t s)} T^{2}
\end{aligned}
$$

and $P$ is pressure, $V$ total volume, $N$ the total number of molecules, $k$ Boltzmann constant, $T$ absolute temperature, $N_{i}$ the number of molecule $i, x_{i}$ the mole fraction of component $i, v_{i}^{(s)}$ the number of group $s$ in a molecule $i, N_{0}$ the number of holes, $N_{T}$ the total number of lattice sites, and $w^{(s)}$ the segment fraction of group $s$. The parameters in the previous model were the unit cell volume $v_{0}$, the number of segments per group $s$ (the number of sites occupied by a group s) $r^{(s)}$, the external degree of freedom per group $s c^{(s)}$, and the potential parameter between $t$ and $s$ segments $U^{(t s)}$. Using parameter values determined from data reduction for pure liquids and solutions, pure liquid properties and liquid mixture properties such as activity coefficients were accurately predicted. However, with regard to saturated vapor pressure the predictions were not satisfactory.

Although there may be a number of reasons for this, a particularly important one may be an inadequacy in the functional form of the equation of state. To examine this possibility, an attempt was made to correlate the saturated properties of methane by Eq. (1) with the values of parameters varied. The methane system was employed here because its molecule can be regarded as composed of a single segment. A good correlation was not obtained over a wide range of temperature.

Such comparisons tended to suggest that the discrepancies might be attributed to the volume dependency of the attraction term in Eq. (1), which was derived from the assumption of a random distribution of segments and holes. Therefore it was considered that the effect of local molecular distribution should be taken into account in the estimation of the potential energy.

According to statistical thermodynamics, an equation of state can be derived from a configurational partition function $Q$ using the following basic equation:

$$
P=k T\left(\frac{\partial \ln Q}{\partial V}\right)_{T, N_{1}, N_{2}, \ldots}
$$

The configurational partition function $Q$ can be approximated by the following form on the basis of lattice theory:

$$
Q=g\left(\prod_{i} v_{f i}^{N_{i}}\right) \exp (-E / k T)
$$


where $g$ is the combinatorial factor, $v_{f i}$ the free volume of molecule $i$, and $E$ the total potential energy.

One successful model which takes into account local molecular distribution is the non-random twoliquid (NRTL) model proposed by Renon and Prausnitz. ${ }^{11)}$ The present work applies this model to the estimation of the potential energy $E$. Accordingly, the local segment fraction of a group $t$ in the nearestneighboring sites surrounding a central segment of group $s, \theta^{(t s)}$, is given as follows:

$$
\theta^{(t s)}=\frac{\theta^{(t)} \eta^{(t s)}}{\theta^{(0)} \eta^{(0 s)}+\sum_{u} \theta^{(u)} \eta^{(u s)}}
$$

where $\theta^{(t)}$ is the fraction of external surface area of the group $t$, and $\eta^{(t s)}$ a parameter characterizing the contribution of segment-pair potential energy to the distribution of segments, given by the following equations:

$$
\begin{gathered}
\theta^{(t)}=\frac{z q^{(t)} y^{(t)}}{z y^{(0)}+\sum_{u} z q^{(u)} y^{(u)}} \\
\eta^{(t s)}=\exp \left\{-\alpha\left(\varepsilon^{(t s)}-\varepsilon^{(s s)}\right) / k T\right\} \\
y^{(t)}=\frac{\sum_{i} N_{i} v_{i}^{(t)}}{N_{0}+\sum_{i} N_{i} \sum_{u} v_{i}^{(u)}}
\end{gathered}
$$

where $z$ is the coordination number, $z q^{(t)}$ the number of external nearest-neighboring sites per group $t, y^{(t)}$ the overall fraction of group $t$ defined by Eq. (16), and $\varepsilon^{t(s)}$ the potential energy between $t$ and $s$ segments. The interactions of both hole-segment and hole-hole pairs, $\varepsilon^{(t 0)}$ and $\varepsilon^{(00)}$, are equal to zero. $\alpha$ is a parameter characterizing the tendency of the segments to distribute in a non-random fashion. If $\alpha=0$, the local segment fractions are equal to the overall segment fractions and the distribution of segments and holes is completely random. According to the concept of local composition, the total potential energy $E$ is given as follows:

$$
\begin{aligned}
E= & \sum_{i} N_{i} E_{i} \\
& E_{i}=\sum_{s} v_{i}^{(s)} E^{(s)} \\
& E^{(s)}=(z / 2) q^{(s)} \sum_{t} \theta^{(t s)} \varepsilon^{(t s)}
\end{aligned}
$$

where $E_{i}$ is the potential energy per molecule $i$ and $E^{(s)}$ the potential energy per group $s$.

It is clear that the combinatorial factor $g$ for the non-random molecular distribution has a smaller value than that calculated from Flory-Huggins' expression adopted in the previous work. However, it is difficult to estimate the effect of the non-randomness on the combinatorial factor quantitatively. Therefore, as the first approximation, Flory-Huggins' expression is used in the present work.* Accordingly,

$$
\begin{aligned}
\ln g= & \sum_{i}\left\{N_{i} \ln \left(\xi_{i} / N_{i}\right)\right\}-N(r-1) \\
& -N_{0} \ln N_{0}+\left(N_{0}+N\right) \ln N_{T}
\end{aligned}
$$

where $\xi_{i}$ is the constant in the Flory-Huggins' approximation.

In the same way as in the previous work, the free volume of molecule $i$ is given as follows:

$$
\begin{gathered}
\ln v_{f i}=\sum_{s} v_{i}^{(s)} r^{(s)} c^{(s)} \ln v_{f, \text { seg }} \\
v_{f, \text { seg }}=v_{0} w^{(0)} \\
v_{0}=V / N_{T} \\
w^{(0)}=N_{0} / N_{T}
\end{gathered}
$$

where $v_{f, \text { seg }}$ is the free volume of a segment, and $w^{(0)}$ the fraction of holes.

From Eqs. (2) through (24), the configurational partition function becomes

$$
\begin{aligned}
\ln Q= & \sum_{i}\left(N_{i} \ln \frac{\xi_{i}}{N_{i}}\right)-N(r-1) \\
& -\frac{V-V_{0} r}{v_{0}} \ln \frac{V-V_{0} r}{v_{0}}+\frac{V-V_{0}(r-1)}{v_{0}} \ln \frac{V}{v_{0}} \\
& -N c \ln \frac{v_{0}\left(V-V_{0} r\right)}{V} \\
& -\frac{1}{T} \sum_{i} N_{i} \sum_{s} \frac{V_{0} v_{i}^{(s)} q^{(s)} U^{(s)}}{\left(V-V_{0} r\right) \eta^{(0 s)}+V_{0} \eta^{(s)}}
\end{aligned}
$$

where

$$
\begin{gathered}
U^{(s)}=(z / 2) \sum_{t} v^{(t)} q^{(t)} \eta^{(t s)} U^{(t s)} \\
U^{(t s)}=\varepsilon^{(t s)} / k \\
\eta^{(s)}=\sum_{t} v^{(t)} q^{(t)} \eta^{(t s)} \\
v^{(t)}=\sum_{i} x_{i} v_{i}^{(t)}
\end{gathered}
$$

An equation of state is derived by substituting Eq. (25) in Eq. (11).

$$
\begin{aligned}
\frac{P V}{N k T}=1 & -r-\frac{V}{V_{0}} \ln \frac{V-V_{0} r}{V}+\frac{c r V_{0}}{V-V_{0} r} \\
& +\frac{1}{T} \sum_{s} \frac{V V_{0} v^{(s)} q^{(s)} U^{(s)} \eta^{(0 s)}}{\left\{\left(V-V_{0} r\right) \eta^{(0 s)}+V_{0} \eta^{(s)}\right\}^{2}}
\end{aligned}
$$

Equation (30) satisfies the ideal gas limit at infinite volume.

* Although Flory-Huggins' equation was derived for athermal solutions, this equation has been applied to fiuids other than athermal solutions. In such a treatment, $\chi$-parameter has been regarded as a term which expresses the effects of entropy as well as enthalpy. In our treatment, the effect of entropy may be included in the potential parameters 
With respect to the free volume, although the previous model employed Eq. (21) following Henderson's hypothesis, another hypothesis was proposed by Sanchez and Lacombe. ${ }^{12)}$ According to the latter hypothesis, the following equation is assumed on the basis of the concept that the effect of the free volume on the repulsive force is expressed by the introduction of holes.

$$
c^{(s)}=0
$$

Since there is not sufficient evidence for either hypothesis to be adopted, the present work has examined both Eq. (21) and (31) as described below.

\section{Correlation of Saturated Properties}

The present model was applied to the $n$-alkane systems from ethane to eicosane. These compounds are made up of methyl and methylene groups. The size of a methylene group is regarded as equal to that of a unit cell and the value of $r^{(s)}$ for the methylene group is taken as unity. The model parameters were determined by reference to the data ${ }^{1,10,15)}$ on vapor pressure and molar densities of vapor and liquid for single-component systems at various temperatures. These calculations were carried out using different values of the parameter $c^{(s)}$. The calculated results indicated a better correlation for $c^{(s)}$-values close to zero. Therefore, Eq. (31) was adopted in the present work for optimization of the parameters.

The values of parameters $U^{(t s)}$ for each group-pair and $v_{0}$ were determined as a function of temperature. From the optimized values at each temperature, it was found that the parameters can be expressed by the following forms:

$$
\begin{gathered}
U^{(t s)}=T_{c}\left\{\dot{C}_{U 1}{ }^{(t s)}+C_{U 2}^{(t s)} / T_{r}+C_{U 3}^{(t s)} / T_{r}^{2}\right\} \\
v_{0}=C_{V 1}\left\{1-C_{V 2} \exp \left(-C_{V 3} T_{r}\right)\right\} \\
T_{r}=T / T_{c}
\end{gathered}
$$

where $T_{c}$ is the critical temperature of the system. The optimized values of the parameters are summarized in Tables 1 and 2 . The fugacity equations used for the calculations are shown in Appendix.

For the sake of convenience the present work has adopted the experimental values for $T_{c}$ for the calculation of $U^{(t s)}$ and $v_{0}$. However, it is possible to calculate $T_{c}$ values by means of Eq. (30), applying the thermodynamic criteria for the critical point. As seen in Fig. 1, the values of $T_{c}$ calculated from the model are in good agreement with the measurements. ${ }^{10)}$ Thus, in a case where critical data are not available, one can calculate the critical values from the model.

The results of correlation of saturated properties are shown in Figs. 2 through 4 . When these results are

\begin{tabular}{|c|c|c|c|}
\hline Group & $r^{(s)}$ & $z q^{(\mathrm{s})}$ & $c^{(s)}$ \\
\hline $\mathrm{CH}_{2}$ & 1 & 0.321 & 0 \\
\hline $\mathrm{CH}_{3}$ & 1.887 & 5.763 & 0 \\
\hline $\begin{array}{c}v_{0} \\
{\left[\mathrm{~cm}^{3} / \mathrm{mol}\right]}\end{array}$ & $\begin{array}{c}C_{Y 1} \\
9.160\end{array}$ & $\begin{array}{c}C_{V 2} \\
0.9442\end{array}$ & $\begin{array}{c}C_{V 3} \\
0.4591\end{array}$ \\
\hline$\alpha$ & 4.029 & & \\
\hline
\end{tabular}
compared with those obtained previously, ${ }^{5}$ it can be seen that vapor pressures and vapor molar densities

\begin{tabular}{|c|c|c|c|}
\hline \multicolumn{2}{|c|}{ Group } & $\mathrm{CH}_{2}$ & $\mathrm{CH}_{3}$ \\
\hline \multirow{3}{*}{$\mathrm{CH}_{2}$} & $C_{U 1}$ & -1.340 & -1.818 \\
\hline & $C_{U 2}$ & 0.1466 & 1.073 \\
\hline & $\mathrm{C}_{U 3}$ & 0.01444 & -0.3188 \\
\hline \multirow{3}{*}{$\mathrm{CH}_{3}$} & $C_{U 1}$ & & -0.6443 \\
\hline & $C_{U 2}$ & & -0.06687 \\
\hline & $C_{U 3}$ & & 0.04583 \\
\hline
\end{tabular}

Table 1. Group parameters

Table 2. Segment-pair potential parameters $U^{(t s)}$

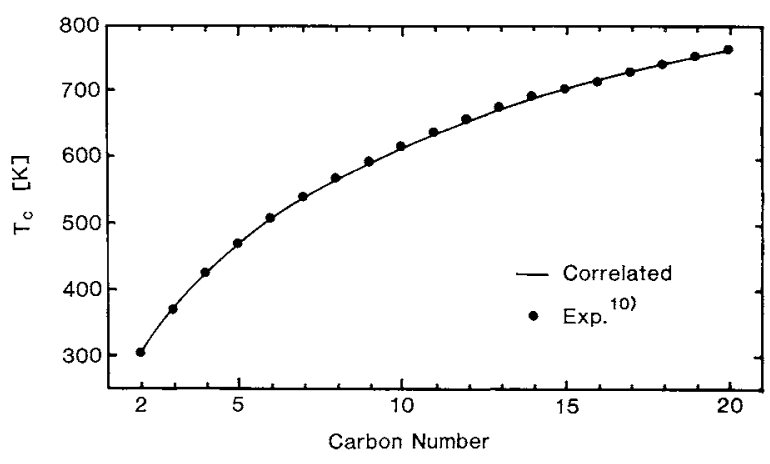

Fig. 1. Critical temperature of $n$-alkanes.

are correlated much better by using the present model. However, one can see in Fig. 4 that there are still some deviations between calculated and experimentally observed liquid molar densities.

\section{Prediction of Vapor-Liquid Equilibria}

The present model has been applied to the prediction of vapor-liquid equilibria for binary mixtures: ethane- $n$-butane and $n$-butane- $n$-decane systems. The parameters $U^{(t s)}$ and $v_{0}$ in Eqs. (32) and (33) were calculated with the following mixing rule for the critical temperatures of the mixtures.

$$
T_{c}=\sum_{i} \sum_{j} x_{i} x_{j}\left(T_{c i} T_{c j}\right)^{1 / 2}
$$

The result for the ethane- $n$-butane system is shown in Fig. 5; the calculated values compare well with the measurements. ${ }^{7)}$ The result for the $n$-butane$n$-decane system is presented in Fig. 6. Although minor discrepancies between experimental and calculated values can be seen, the model does predict values which are close to the measurements. ${ }^{9)}$ 


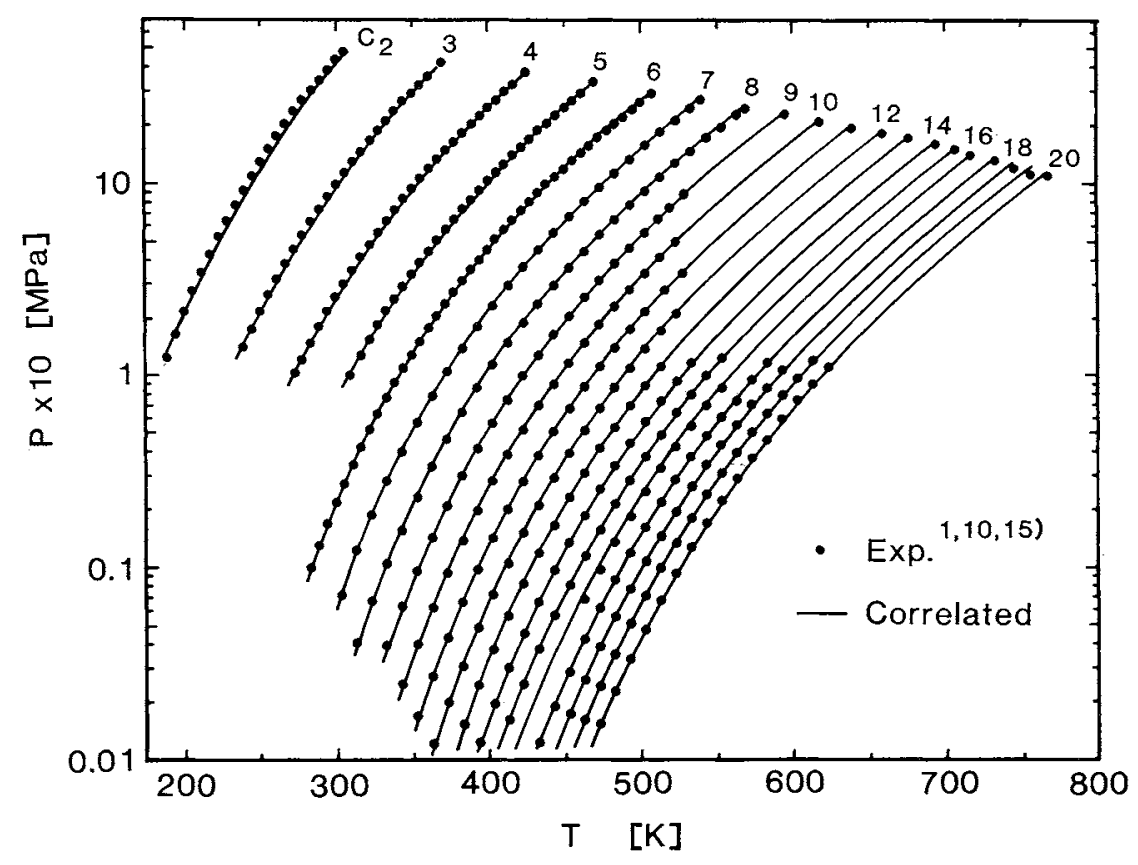

Fig. 2. Saturated vapor pressure of $n$-alkanes.

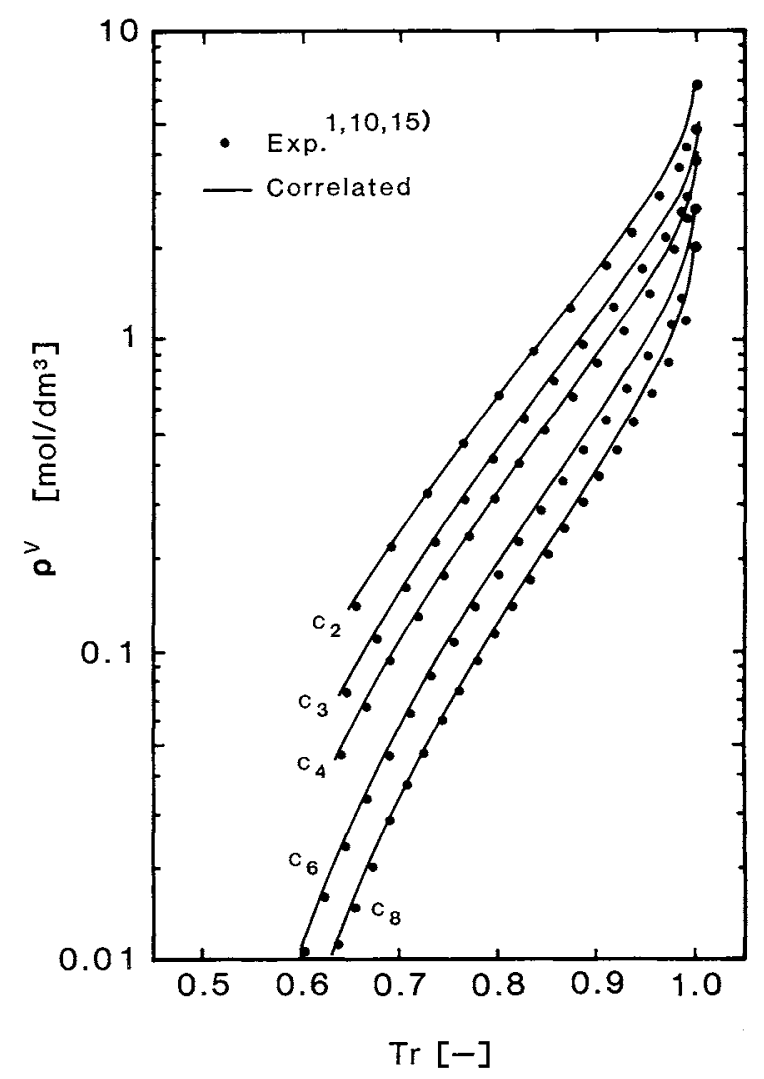

Fig. 3. Saturated molar density of vapor phase for $n$ alkanes.

\section{Conclusion}

A new equation of state is derived from a group contribution model which takes into account the effect of non-random molecular distribution on molecular potential energy. The ability of the model to

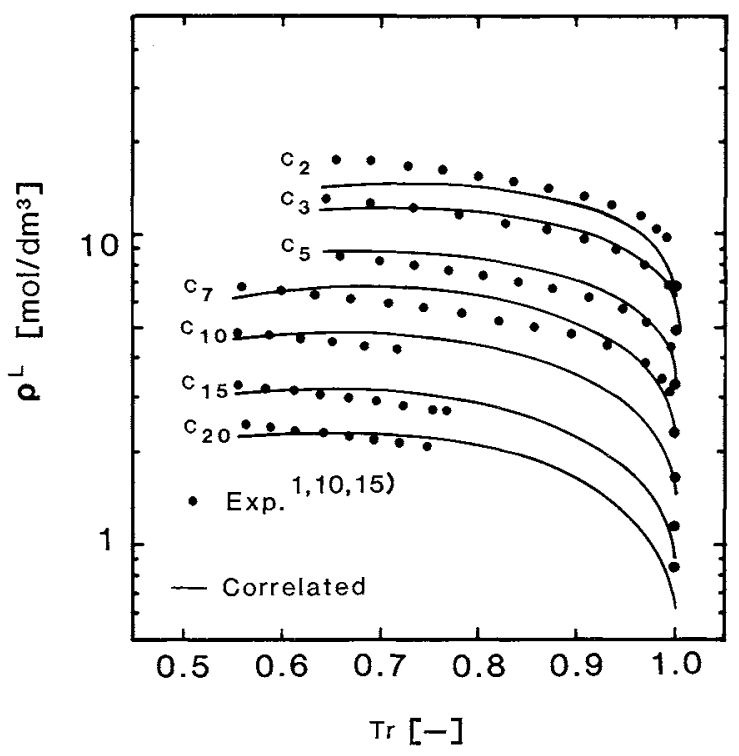

Fig. 4. Saturated molar density of liquid phase for $n$ alkanes.

correlate saturated properties is shown by a comparison with experimental data for $n$-alkanes. An attempt is made to examine the applicability of the model to the prediction of vapor-liquid equilibria for binary mixtures. Good agreement is obtained between calculated and experimental values for the ethane- $n$ butane system. For the $n$-butane- $n$-decane system, the prediction is not yet satisfactory, but the model does predict values close to the experimental data.

Appendix: Equations of fugacity

Equations of fugacity are obtained from Eq. (30). The fugacity equation for a pure system is given as follows: 


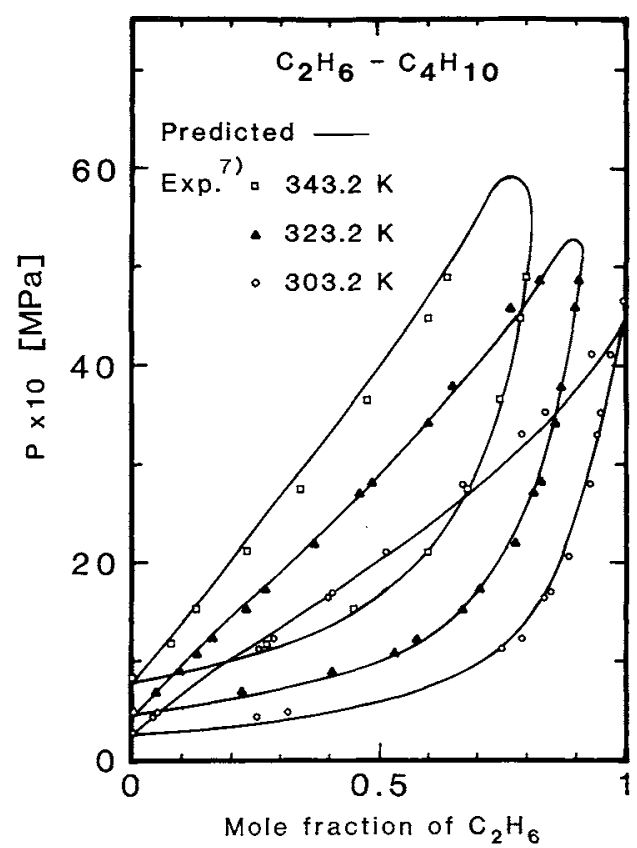

Fig. 5. Vapor-liquid equilibria for ethane- $n$-butane system.

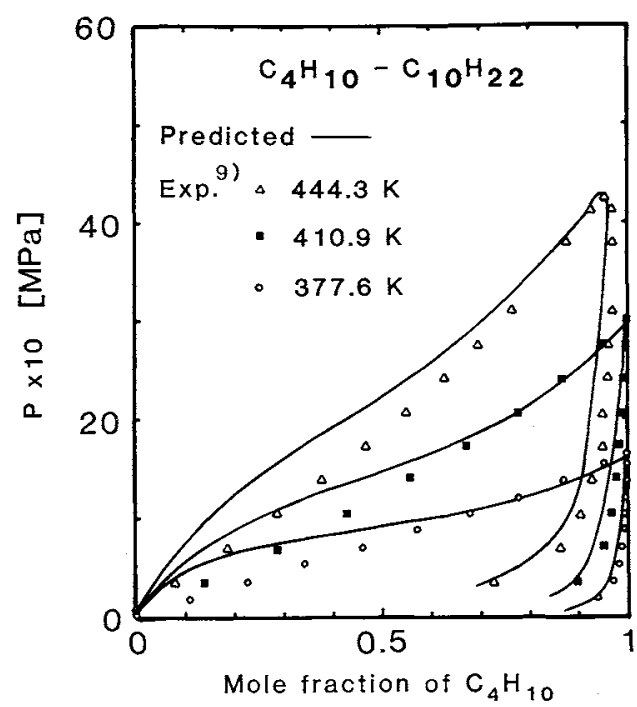

Fig. 6. Vapor-liquid equilibria for $n$-butane- $n$-decane system.

$$
\begin{aligned}
\ln \frac{f}{P}= & r+\left(\frac{V}{V_{0}}-r-c\right) \ln \frac{V-V_{0} r}{V} \\
& +\frac{1}{T} \sum_{s} \frac{V_{0} v^{(s)} q^{(s)} U^{(s)}}{\left(V-V_{0} r\right) \eta^{\left(0_{s}\right)}+V_{0} \eta^{(s)}}-\ln Z+Z-1
\end{aligned}
$$

where $Z$ is the compressibility factor. For a mixture the fugacity of a component $i$ is expressed as follows:

$$
\begin{aligned}
\ln \frac{f_{i}}{x_{i} P}= & -\left(r_{i}+c_{l}\right) \ln \frac{V-V_{0} r}{V} \\
& -\frac{n}{v_{0}}\left(\frac{\partial v_{0}}{\partial n_{i}}\right)\left\{\frac{V}{V_{0}} \ln \frac{V-V_{0} r}{V}+r\right\}+\frac{3 c n}{V-V_{0} r}\left(\frac{\partial V_{0} r}{\partial n_{i}}\right) \\
& +\frac{1}{T} \sum_{s} \frac{B}{A}\left[\frac{v_{i}^{(s)}}{v^{(s)}}+\frac{n}{V_{0} U^{(s)} \eta^{(0 s)}}\left(\frac{\partial V_{0} U^{(s)} \eta^{(0 s)}}{\partial n_{i}}\right)-\frac{2 n}{\eta^{(0 s)}}\left(\frac{\partial \eta^{(0 s)}}{\partial n_{i}}\right)\right. \\
& \left.+\frac{1}{A}\left\{n\left(\frac{\partial V_{0} r}{\partial n_{i}}\right)+\frac{V_{0} \eta^{(s)}}{\left(\eta^{(0 s)}\right)^{2}} n\left(\frac{\partial \eta^{(0 s)}}{\partial n_{i}}\right)-\frac{n}{\eta^{(0 s)}}\left(\frac{\partial V_{0} \eta^{(s)}}{\partial n_{i}}\right)\right\}\right]-\ln Z
\end{aligned}
$$

where

$$
\begin{aligned}
& A=\left(V-V_{0} r\right) \eta^{(0 s)}+V_{0} \eta^{(s)} \\
& B=V_{0} v^{(s)} q^{(s)} U^{(s)} \\
& n\left(\frac{\partial v_{0}}{\partial n_{i}}\right)=-C_{V 1} C_{V 2} C_{V 3} \exp \left(-C_{V 3} T_{r}\right) n\left(\frac{\partial T_{r}}{\partial n_{i}}\right) \\
& n\left(\frac{\partial T_{r}}{\partial n_{i}}\right)=2 T_{r} \frac{T_{c}^{1 / 2}-T_{c i}^{1 / 2}}{T_{c}^{1 / 2}} \\
& \left(\frac{\partial V_{0} r}{\partial n_{i}}\right)=v_{0} r_{i}+r n\left(\frac{\partial v_{0}}{\partial n_{i}}\right) \\
& \left(\frac{\partial V_{0}}{\partial n_{i}}\right)=v_{0}+n\left(\frac{\partial v_{0}}{\partial n_{i}}\right) \\
& \left(\frac{\partial V_{0} U^{(s)} \eta^{(0 s)}}{\partial n_{i}}\right)=U^{(s)} \eta^{(0 s)}\left(\frac{\partial V_{0}}{\partial n_{i}}\right)+v_{0} \eta^{(0 s)} n\left(\frac{\partial U^{(s)}}{\partial n_{i}}\right)+v_{0} U^{(s)} n\left(\frac{\partial \eta^{(0 s)}}{\partial n_{i}}\right) \\
& \left(\frac{\partial V_{0} \eta^{(s)}}{\partial n_{i}}\right)=\eta^{(s)}\left(\frac{\partial V_{0}}{\partial n_{i}}\right)+v_{0} n\left(\frac{\partial \eta^{(s)}}{\partial n_{i}}\right) \\
& { }_{n}\left(\frac{\partial U^{(s)}}{\partial n_{i}}\right)=-U^{(s)}+\frac{z}{2} \sum_{t} v_{i}^{(t)} q^{(t)} \eta^{(t s)} U^{(t s)}+\frac{z}{2} \sum_{t} v^{(t)} q^{(t)} n\left(\frac{\partial \eta^{(t s)} U^{(2 s)}}{\partial n_{i}}\right) \\
& n\left(\frac{\partial \eta^{(s)} U^{(t s)}}{\partial n_{i}}\right)=\eta^{(t s)} n\left(\frac{\partial U^{(t s)}}{\partial n_{i}}\right)+U^{(t s)} n\left(\frac{\partial \eta^{(t s)}}{\partial n_{i}}\right) \\
& n\left(\frac{\partial \eta^{(s)}}{\partial n_{i}}\right)=-\eta^{(s)}+\sum_{t} v_{i}^{(t)} q^{(t)} \eta^{(t s)}+\sum_{t} y^{(t)} q^{(t)} n\left(\frac{\partial \eta^{(t s)}}{\partial n_{i}}\right) \\
& n\left(\frac{\partial U^{(t s)}}{\partial n_{i}}\right)=-\frac{1}{T_{r}}\left\{U^{(t s)}+\frac{T_{c}}{T_{r}}\left(C_{U 2}^{(t s)}+\frac{2 C_{U 3}^{(t s)}}{T_{r}}\right)\right\} n\left(\frac{\partial T_{r}}{\partial n_{i}}\right) \\
& n\left(\frac{\partial \eta^{(t s)}}{\partial n_{i}}\right)=\frac{\alpha \eta^{(t s)}}{T}\left\{n\left(\frac{\partial U^{(t s)}}{\partial n_{i}}\right)-n\left(\frac{\partial U^{(s s)}}{\partial n_{i}}\right)\right\}
\end{aligned}
$$

\begin{tabular}{|c|c|c|}
\hline$c$ & $=$ external degree of freedom for mixture & {$[-$} \\
\hline$c_{i}$ & $=$ external degree of freedom per molecule $i$ & \\
\hline$c^{(s)}$ & $=$ external degree of freedom per group $s$ & \\
\hline$E$ & $=$ total potential energy & \\
\hline$E_{i}$ & $=$ potential energy per molecule $i$ & \\
\hline$E^{(s)}$ & $=$ potential energy per group $s$ & \\
\hline$f$ & $=$ fugacity of a pure component & {$[\mathrm{M}]$} \\
\hline$f_{i}$ & $=$ fugacity of component $i$ in a mixture & {$[N$} \\
\hline$g$ & $=$ combinatorial factor & \\
\hline$k$ & $=$ Boltzmann's constant & {$[\mathrm{erg} /$} \\
\hline$N$ & $=$ total number of molecules & \\
\hline$N_{T}$ & $=$ total number of lattice sites & \\
\hline$N_{i}$ & $=$ number of molecule $i$ & \\
\hline$N_{0}$ & $=$ number of holes & \\
\hline$n$ & $=$ total mole number & {$[\mathrm{m}$} \\
\hline$n_{i}$ & $=$ mole number of component $i$ & \\
\hline$P$ & $=$ pressure & {$[\mathrm{MF}$} \\
\hline$P_{s}$ & $=$ saturated vapor pressure & {$[\mathrm{M}$} \\
\hline$Q$ & $=$ configurational partition function & \\
\hline$q^{(s)}$ & $=$ parameter of external surface area of gr & \\
\hline & $=$ number of segments for mixture & \\
\hline
\end{tabular}

\section{Acknowledgment}

This work was supported in part by Grant-in-Aid for Scientific Research (No. 58430017) from the Ministry of Education, Science and Culture of Japan in 1984, for which the authors express their thanks.

\section{Nomenclature}




\begin{tabular}{|c|c|c|}
\hline$r_{i}$ & $=$ number of segments per molecule $i$ & {$[-]$} \\
\hline$r^{(s)}$ & $=$ number of segments per group $s$ & {$[-]$} \\
\hline$T$ & $=$ absolute temperature & {$[\mathrm{K}]$} \\
\hline$T_{c}$ & $=$ critical temperature & {$[\mathrm{K}]$} \\
\hline$T_{r}$ & $=$ reduced temperature & {$[-]$} \\
\hline$T^{*}$ & $=$ parameter in Eq. (1), (see ref. 5) & {$[\mathrm{K}]$} \\
\hline$U^{(t s)}$ & $=$ potential parameter of $t-s$ segment pair & {$[\mathrm{K}]$} \\
\hline$V$ & $=$ total volume & {$\left[\mathrm{cm}^{3}\right]$} \\
\hline$v_{0}$ & $=$ unit cell volume & {$\left[\mathrm{cm}^{3}\right]$} \\
\hline$v_{f i}$ & $=$ free volume of molecule $i$ & {$\left[\mathrm{~cm}^{3}\right]$} \\
\hline$V_{f, \operatorname{seg}}$ & $=$ free volume of a segment & {$\left[\mathrm{cm}^{3}\right]$} \\
\hline$w^{(s)}$ & $=$ segment fraction of groups $s$ & {$[-]$} \\
\hline$w^{(0)}$ & $=$ fraction of holes & {$[-]$} \\
\hline$x_{i}$ & $=$ mole fraction of component $i$ & {$[-]$} \\
\hline$y^{(t)}$ & $=$ fraction of group $t$ & {$[-]$} \\
\hline$Z$ & $=$ compressibility factor & {$[---]$} \\
\hline$z$ & $=$ coordination number & {$[-]$} \\
\hline$\alpha$ & $=$ parameter in Eq. (15) & {$[-]$} \\
\hline$\varepsilon^{(t s)}$ & $=$ potential energy of $t-s$ segment pair & [erg] \\
\hline$\eta^{(t s)}$ & $\begin{array}{l}=\text { parameter characterizing the non-random } \\
\text { distribution of segments }\end{array}$ & {$[-]$} \\
\hline$\theta^{(t)}$ & $=$ fraction of external surface area of group $t$ & {$[-]$} \\
\hline$\theta^{(t s)}$ & $\begin{array}{l}=\text { local segment fraction of group } t \text { in the } \\
\text { immediate vicinity of group } s\end{array}$ & {$[-]$} \\
\hline$v_{i}^{(s)}$ & $=$ number of group $s$ in a molecule $i$ & {$[-]$} \\
\hline & $=$ constant in Flory-Huggins' approximation & {$[-]$} \\
\hline$\rho^{L}$ & $=$ saturated molar density of liquid phase [mo & $\left.1 / \mathrm{dm}^{3}\right]$ \\
\hline$\rho^{r}$ & $=$ saturated molar density of vapor phase [mo & $\left.1 / \mathrm{dm}^{3}\right]$ \\
\hline
\end{tabular}

Literature Cited

1) Canjar, L. N. and F. S. Manning: "Thermodynamic
Properties and Reduced Correlations for Gases," Gulf Pub. Co., Houston, Texas (1967).

2) Flory, P. J.: J. Chem. Phys., 9, 660 (1941); 10, 51 (1942).

3) Henderson, D.: J. Chem. Phys., 37, 631 (1962).

4) Huggins, M. L.: J. Phys. Chem., 9, 440 (1941).

5) Ishizuka, I., E. Sarashina, Y. Arai and S. Saito: J. Chem. Eng. Japan, 13, 90 (1980).

6) Lee, T. W., R. A. Greenkorn and K. C Chao: Ind. Eng. Chem., Fundam., 11, 293 (1972); Chem. Eng. Sci., 28, 1005 (1973).

7) Lhotac, V. and I. Wichterle: Fluid Phase Equilibria, 6, 229 (1981).

8) Nitta, T., E. A. Turek, R. A. Greenkorn and K. C. Chao: AIChE J., 23, 144 (1977).

9) Reamer, H. H. and B. H. Sage: J. Chem. Eng. Data, 9, 24 (1964).

10) Reid, R. C., J. M. Prausnitz and T. K. Sherwood: "The Properties of Gases and Liquids," 3rd Ed., McGraw-Hill (1977).

11) Renon, H. and J. M. Prausnitz: AIChE J., 14, 135 (1968).

12) Sanchez, I. C. and R. H. Lacombe: J. Phys. Chem., 80, 2352 (1976).

13) Skjold-Jørgensen, S.: Fluid Phase Equilibria, 16, 317 (1984).

14) Tochigi, K., K. Kurihara and K. Kojima: J. Chem. Eng. Japan, 18, 60 (1985).

15) Vargaftic, N. B.: "Tables on the Thermophysical Properties of Liquids and Gases," 2nd Ed., Hemisphere Pub. Co., Washington, D.C. (1975).

\title{
RELATION BETWEEN PRESSURE LOSS AND COLLECTED PARTICLE WEIGHTS IN A HIGH GRADIENT MAGNETIC SEPARATOR (HGMS)
}

\author{
HARUMI MATSUZAKI, MASAYOSHI KUBOTA, SANKICHI TAKAHASHI \\ KATSUYA EBARA AND TOSHIMI MUKUSHI \\ Hitachi Research Laboratory, Hitachi Ltd., Hitachi 317
}

Key Words: High Gradient Magnetic Separator, Metallic Particles, Fiber Matrix, Chip Matrix, Pressure
Loss, Collected Particle Weight

\section{Introduction}

A number of studies ${ }^{2,3,5-9)}$ have appeared on the particle collection mechanism in a HGMS. But little work $^{1,4)}$ has been done on the relation between pressure loss in a matrix and the collected particle weights on fillings.

\footnotetext{
Received December 10, 1984. Correspondence concerning this article should be addressed to $\mathrm{H}$. Matsuzaki.
}

In this report, the collection characteristics of fine metallic particles with paramagnetism were studied from the latter viewpoint, using a HGMS with either a fiber or a chip matrix.

\section{Experimental Apparatus and Method}

The apparatus for the experiments is shown in Fig. 1. The HGMS had a matrix $8 \mathrm{~mm}$ deep located between magnetic poles $80 \mathrm{~mm}$ in diameter. The 